\title{
Some Notes on the Proof of Entropy Increase in the Thermalization of Two Blocks
}

\section{Li Pinjun}

Department of Information Science, Zhanjiang Preschool Education College, Zhanjiang, China

Email address:

pingyuejushi@126.com

To cite this article:

Li Pinjun. Some Notes on the Proof of Entropy Increase in the Thermalization of Two Blocks. American Journal of Physics and Applications. Vol. 9, No. 1, 2021, pp. 10-14. doi: 10.11648/j.ajpa.20210901.12

Received: October 14, 2020; Accepted: December 10, 2020; Published: January 22, 2021

\begin{abstract}
The thermalization of two blocks with different initial temperatures in an insulated recipient is an irreversible process, so the entropy of the system will increase during this process. Lima (Eur. J. Phys. 36 (2015) 068001) has given an elegant and concise proof for that which had been proved by Mungan (Eur. J. Phys. 36 (2015) 048004) with a complex method. However, there are still two problems in Lima's proof: 1. It is assumed that the heat capacities of two blocks are constants, which is not true in most practical cases. 2. An inequality that describes the concavity of the logarithm function was used but it is still relatively uncommon for beginners. In this article, two stricter and simpler proof were given for the problem 1 by making use of $1 / \mathrm{T}-\mathrm{Q}$ diagram and T-S diagram, respectively. In the Proof by $1 / T-\mathrm{Q}$ diagram, the area under the curve of $1 / T$ over the domain $\left[0, \mathrm{Q}_{0}\right]$ is the value of the entropy change of the cooler block, which is positive; while the area under the curve of $1 / T$ ' over the domain $\left[\mathrm{Q}_{0}, 0\right]$ is the value of the entropy change of the hotter block, which is negative. It is rather intuitive to compare these two values by using the monotonicity and domains of $T$ and $T$ '. A similar method is adopted in the proof by T-S diagram. For the problem 2, another proof for the key inequality in Mungan's paper was given by using elementary geometric method which is really more suitable for physics beginners.
\end{abstract}

Keywords: Thermalization, Principle of Entropy Increase, Second Law of Thermodynamics

\section{Introduction}

The thermalization of two blocks with different initial temperatures in an insulated recipient is an irreversible process. According to the second law of thermodynamics, the entropy of the system will increase (the sum of the entropy changes of the two blocks is greater than zero). It may be due to the difficulty or length, there is no proof for that in the existing textbooks [1-15]. Mungan and Lima successively presented their proofs in reference [16, 17]. Mungan's proof feels rather complicated, which was improved by Lima, whose proof is concise and elegant. However, there are still two problems in it: 1 . It is assumed that the heat capacity of the blocks are constants, which is not true in most practical cases. 2. An inequality that describes the concavity of the logarithm function was used but this inequality is still relatively uncommon for beginners. In this article, a stricter and simpler proof was given for the first problem making use of Q-1/T diagram and T-S diagram respectively. For the second problem, another proof for the key inequality in Mungan's paper was given by using elementary geometric method which is really more suitable for physics beginners.

\section{Brief Description of the Problem}

Suppose there are two blocks 1 and 2 in an insulated recipient, their initial temperatures are $T_{1}$ and $T_{2}$ (without loss of generality, assume that $T_{1}>T_{2}>0$ ), and their heat capacities are $C_{1}$ and $C_{2}$. When they are brought to contact each other, they exchange energy as heat and come to a final state called equilibrium state, both with the same temperature $T_{e q}\left(T_{1}>T_{e q}>T_{2}\right)$.

When calculating the entropy change of the system, a quasi-static process needs to be set up, for example, two temperature-controlled heat sources are used to contact the two blocks respectively [2], so that their temperature slowly 
decreases or rises to $T_{e q}$. In this process, the entropy changes of block 1 and 2 are:

$$
\Delta S_{1}=\int \frac{d Q}{T}
$$

and

$$
\Delta S_{2}=\int \frac{d Q^{\prime}}{T^{\prime}}
$$

respectively.

The variable $T\left(T^{\prime}\right)$ in the formula is the real-time temperature of the block 1 (2), its value gradually decreases (increases) from $T_{1}\left(T_{2}\right)$ to $T_{e q}, d Q=\mathrm{C}_{1} d T\left(d Q^{\prime}=\mathrm{C}_{2} d T^{\prime}\right)$ is the heat released (absorbed) by block 1 (2) when its temperature changes from $T\left(T^{\prime}\right)$ to $T+d T\left(T^{\prime}+d T^{\prime}\right)$, which is a negative (positive) value. In most textbooks and literatures, it is assumed that the heat capacities $C_{1}$ and $C_{2}$ are constants (which is often not the case in practice). Substituting $d Q=\mathrm{C}_{1} d T$ and $d Q^{\prime}=\mathrm{C}_{2} d T^{\prime}$ into equations (1) and (2) respectively and integrating them can obtain the total entropy change of the system [2]

$$
\Delta S=\Delta S_{1}+\Delta S_{2}=\ln \left(\frac{C_{1} T_{1}+C_{2} T_{2}}{T_{1}\left(C_{1}+C_{2}\right)}\right)^{C_{1}}+\ln \left(\frac{C_{1} T_{1}+C_{2} T_{2}}{T_{2}\left(C_{1}+C_{2}\right)}\right)^{C_{2}}
$$

According to the second law of thermodynamics, it should be greater than zero. That is

$$
\ln \left(\frac{C_{1} T_{1}+C_{2} T_{2}}{T_{1}\left(C_{1}+C_{2}\right)}\right)^{C_{1}}+\ln \left(\frac{C_{1} T_{1}+C_{2} T_{2}}{T_{2}\left(C_{1}+C_{2}\right)}\right)^{C_{2}}>0
$$

At present, no strict proof of the formula above has been found in the available textbooks, and most of them are only in the form of special cases (such as the equality of $C_{1}$ and $C_{2}$, or one of the two blocks is a constant temperature heat source, or give specific heat capacities and initial temperature values, etc.) to verify the formula. However, verification is not proof after all, because it is not arbitrary. In order to prove that formula (4) is true, here are some equivalent transformations:

$$
\begin{gathered}
\Delta S \geq 0 \Leftrightarrow \ln \left(\frac{C_{1} T_{1}+C_{2} T_{2}}{T_{1}\left(C_{1}+C_{2}\right)}\right)^{C_{1}}+\ln \left(\frac{C_{1} T_{1}+C_{2} T_{2}}{T_{2}\left(C_{1}+C_{2}\right)}\right)^{C_{2}} \geq 0 \\
\Leftrightarrow \ln \left(\frac{C_{1} T_{1}+C_{2} T_{2}}{T_{2}\left(C_{1}+C_{2}\right)}\right)^{C_{2}} \geq-\ln \left(\frac{C_{1} T_{1}+C_{2} T_{2}}{T_{1}\left(C_{1}+C_{2}\right)}\right)^{C_{1}}=\ln \left(\frac{T_{1}\left(C_{1}+C_{2}\right)}{C_{1} T_{1}+C_{2} T_{2}}\right)^{C_{1}} \\
\Leftrightarrow\left(\frac{C_{1} T_{1}+C_{2} T_{2}}{T_{2}\left(C_{1}+C_{2}\right)}\right)^{C_{2}} \geq\left(\frac{T_{1}\left(C_{1}+C_{2}\right)}{C_{1} T_{1}+C_{2} T_{2}}\right)^{C_{1}} \text { (Here I make use of }
\end{gathered}
$$

the property that the function $\ln x$ is a monotone increasing function.)

$$
\Leftrightarrow\left(\frac{C_{1} T_{1}+C_{2} T_{2}}{C_{1} T_{2}+C_{2} T_{2}}\right)^{C_{2}} \geq\left(\frac{C_{1} T_{1}+C_{2} T_{1}}{C_{1} T_{1}+C_{2} T_{2}}\right)^{C_{1}} \Leftrightarrow\left(\frac{C_{1} T_{1}+C_{2} T_{2}}{C_{1} T_{2}+C_{2} T_{2}}\right)^{\frac{C_{2}}{C_{1}}} \geq \frac{C_{1} T_{1}+C_{2} T_{1}}{C_{1} T_{1}+C_{2} T_{2}}
$$

$$
\Leftrightarrow\left(\frac{\frac{T_{1}}{T_{2}}+\frac{C_{2}}{C_{1}}}{1+\frac{C_{2}}{C_{1}}}\right)^{\frac{C_{2}}{C_{1}}} \geq \frac{\frac{T_{1}}{T_{2}}+\frac{C_{2}}{C_{1}} \frac{T_{1}}{T_{2}}}{\frac{T_{1}}{T_{2}}+\frac{C_{2}}{C_{1}}} \text { (In this step, both the }
$$

numerator and denominator were divided by $C_{1} T_{2}$ ).

(Now let $\frac{C_{2}}{C_{1}}=r-1$, Then $\mathrm{r}$ must be greater than 1 , namely $0<\frac{1}{r}<1 ;$ let $\frac{T_{1}}{T_{2}}=\gamma+1$, then $\gamma+1>0$ ).

$$
\Leftrightarrow\left(\frac{r+\gamma}{r}\right)^{r-1} \geq \frac{r \gamma+r}{r+\gamma} \text { (in this step, the number of the }
$$
variables in the inequality were reduced to two).

$$
\Leftrightarrow\left(\frac{r+\gamma}{r}\right)^{r-1} \geq \frac{r \gamma+r}{r+\gamma}=\frac{r(\gamma+1)}{r+\gamma}
$$

$\Leftrightarrow\left(\frac{r+\gamma}{r}\right)^{r-1} \frac{(r+\gamma)}{r} \geq \gamma+1 \quad$ (According to the settings above, $r+\gamma>0$.)

$$
\begin{gathered}
\Leftrightarrow\left(\frac{r+\gamma}{r}\right)^{r} \geq \gamma+1 \\
\Leftrightarrow\left(1+\frac{1}{r} \gamma\right)^{r} \geq 1+\gamma \\
\Leftrightarrow r \ln \left(1+\frac{1}{r} \gamma\right) \geq \ln (1+\gamma) \\
\Leftrightarrow \ln \left(1+\frac{1}{r} \gamma\right)>\frac{1}{r} \ln (1+\gamma)
\end{gathered}
$$

After that, Mungan expanded the left side of inequality (5) with binomial theorem and proved the inequality on this basis. His proof is as troublesome as Lima pointed out. Lima made use a property of concavity of the logarithm function, namely

$$
\ln (t a+(1-t) b)>t \ln a+(1-t) \ln b \quad(\text { for all } 0<t<1) \text { (7) }
$$

in any open interval $(a, b)$ to prove inequality (6)[17]. His proof is concise and elegant. However, inequality (7) is still relatively uncommon for beginners.

\section{Mistakes That Are Easy to Make and Two New Methods of Proof}

\subsection{Mistakes That Are Easy to Make}

Some literatures believe that in the process of thermalization between two blocks, whenever the block 1 at temperature $T$ transfers a small amount of heat $\mathrm{d} Q$ to the block 2 at temperature $T^{\prime}$, the entropy change of the system is (note: 
$\left.T>T^{\prime}\right)$

$$
d S=\frac{-d Q}{T}+\frac{d Q}{T^{\prime}}>0
$$

In that case, the entropy change of the whole process must be greater than zero. This idea is incorrect in principle, because the direct thermalization between two blocks whose initial temperature difference is not infinitesimal is an irreversible process and therefore cannot be used to calculate the entropy change. Now I give the other two proofs which are rather simple and strict below.

\subsection{The Proof by $1 / T-Q$ Diagram}

In the quasi-static process set in reference [2], the block 1 transferred heat $\mathrm{Q}_{0}$ to the temperature controllable heat source very slowly and continuously, so formula (1) can be more accurately expressed as

$$
\Delta S_{1}=\int_{0}^{-Q_{0}} \frac{d Q}{T}=-\int_{0}^{Q_{0}} \frac{1}{T} d Q<0
$$

Similarly, formula (2) can be expressed as

$$
\Delta S_{2}=\int_{0}^{Q_{0}} \frac{1}{T^{\prime}} d Q^{\prime}>0
$$

Let us consider $1 / T$ and $1 / T^{\prime}$ as some kind of weight or distribution function with respect to $Q$ and $Q^{\prime}$ respectively. Since the change of $T$ and $T^{\prime}$ are monotonous and continuous, and the minimum value of $T$ is equal to the maximum value of $T^{\prime}$ ( That is to say, the integral weights in formula (10) are significantly larger than the integral weights in formula (9) except at the balance point where their weights are equal), it is obvious that the absolute value of $\Delta S_{1}$ is less than the

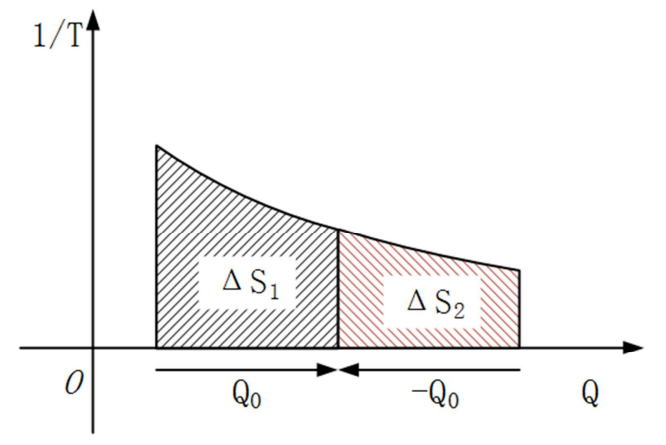

a. Because the variation width of the abscissa that represent the heat absorbed by the initially colder block is equals to the variation width that represent the heat released by the hotter block, and the function $1 / \mathrm{T}$ decreases monotonously, the shadow area $\Delta \mathrm{S}_{1}$ which is positive is larger than the shadow area $\Delta S_{2}$ which is negative. absolute value of $\Delta S_{2}$, so their sum, that is, the entropy change of the system must be greater than zero. Since this proof does not involve the heat capacities of the blocks, it is also true for cases where the heat capacities are not constants.

The proof process above actually corresponds to an intuitive image of integral (see figure 1a). The process may be represented for each block in the 1/T-Q diagram by a column with height given by $1 / \mathrm{T}$ and width indicating the heat quality variation (namely $\mathrm{Q}_{0}$ ) for the considered block. In addition, the greatest height $1 / \mathrm{T}$ (eq) for the initially hotter block is equal to the smallest height for the initially colder block, and the area under these curves are the entropy change of the two blocks respectively. Thus, one must conclude that the area of the column of the initially colder block (corresponding to the entropy variation of this block) must be larger than that for the initially hotter block (note that $\mathrm{Q}\{$ cold $\}=-\mathrm{Q}\{$ hot $\}=\mathrm{Q}_{0}$ ).

\subsection{Another Equivalent Proof by T-S Diagram}

One interesting proof can be provided by using the temperature-entropy diagram (see figure $1 \mathrm{~b}$ ). Its essence is the same as above, both make use of the heat absorbed by the initially colder block equals to the heat emitted by the initially hotter block, in the meanwhile, the temperature of both is monotonous and continuous. The quasi-static process may be represented for each block in the T-S diagram by a column with height given by $\mathrm{T}$ and width indicating the entropy variation (namely $\mathrm{dS}$ ) for the considered block. In addition, the greatest height $\mathrm{T}(\mathrm{eq})$ for the initially colder block is equal to the smallest height for the initially hotter block, and the area under these curves must be the same due to energy balance (note that $\mathrm{Q}\{$ cold $\}=-\mathrm{Q}\{$ hot $\}$ yet). Thus, one must conclude that the width of the column of the initially colder block (corresponding to the entropy variation of this block) must be larger than that for the initially hotter block.

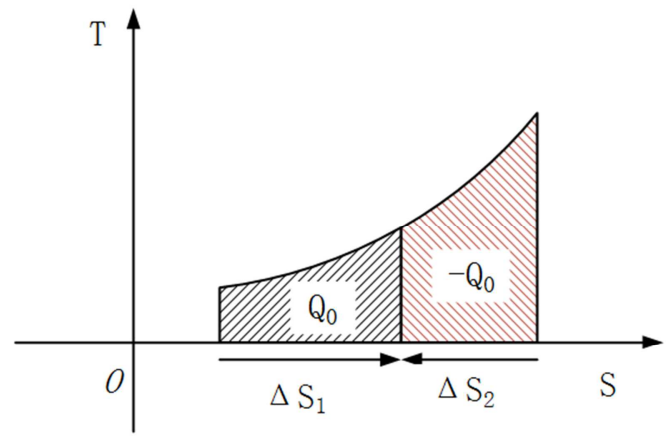

b. Because $\mathrm{T}$ is monotonously increasing with respect to $\mathrm{Q}$ and the two shadow areas which represent the heat absorbed by the initially colder block and the heat released by the initially hotter block respectively are equal, so the entropy change width of the former which is positive is larger than that of the latter which is negative.

Figure 1. 1/T-Q diagram and T-S diagram. 


\section{Another proof of Inequality (6) (Geometric Method)}

In teaching, it has been found that many students were still interested in the proof of inequality (5) or (6). In order to meet the learning enthusiasm of beginners for mathematical derivation, I provide a geometric method below to prove inequality (6) (still based on the concavity of the function).

To prove the validity of the inequality, the logarithmic function $y=\ln x$ curve is shown in figure 2. For any $\gamma>0$, we can always make a straight line $P Q$ intersecting function curve at point $F$ and make the distance from point $B$ (the projection of $F$ on the $x$ axis) to point $P$ equal to $\gamma$ (i.e. $|P B|=\gamma$ ), so that the abscissa of point $B$ is $\gamma+1$, and the ordinate of point $F$ (i.e. height $|B F|)$ is $\ln (1+\gamma)$. And because $0<\frac{1}{r}<1$, for any $r$, we can always find a point $A$ between $P$ and $B$, so that $|P A|=\frac{1}{r}|P B|=\frac{1}{r} \gamma$, that is, the abscissa of point $A$ is $1+\frac{1}{r} \gamma$. In this way, the ordinate of point $E$ (i.e. $|A E|$ ) on the function curve is $\ln \left(1+\frac{1}{r} \gamma\right)$. It can be obtained from the similarity between $\triangle$ PCA and $\triangle$ PFB that

$$
|A C|=\frac{|P A|}{|P B|}|F B|=\frac{1}{r} \ln (1+\gamma)
$$

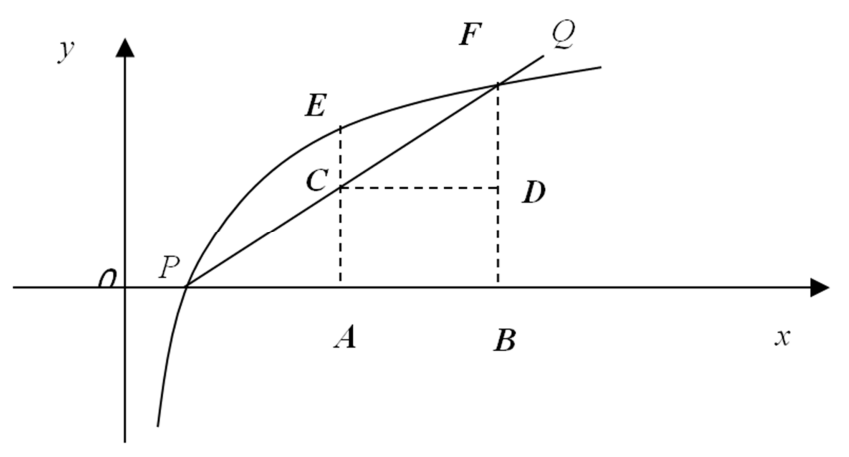

Figure 2. $y=\ln x$ curve.

As can be seen from figure 2, the $y=\ln x$ function curve is concave (this can also be proved based on the relevant knowledge of calculus, which is abbreviated here), so. In this way, we prove the correctness of inequality (6).

\section{Conclusion}

For the proof of the entropy increase in the thermalization of two blocks, it is easy to make the mistake of considering that the entropy change of the system is $d S=-d Q / T+d Q / T^{\prime}$ in a short period of time. It should be noted that the entropy-change of irreversible processes cannot be calculated by the ratio of heat to temperature. In this paper, an appropriate quasi-static process is selected. Without changing $\mathrm{d} Q, 1 / \mathrm{T}$ and $1 / T^{\prime}$ are regarded as some kind of weight or distribution function about $Q$ and $Q^{\prime}$ respectively, which can make the proof very simple and more rigorous. For the entropy change presented by Mungan and Lima as the result of integration, it can be proved to be positive by the geometric method using the concavity of the function, which is more intuitive and easier to understand.

\section{Acknowledgements}

This work was sponsored by Special Funds for the "Climbing" Program of Guangdong Province (Key Items) (No. pdjh2020a1315).

\section{References}

[1] Hugh D. Young, Roger Freedman, A. Lewis Ford. Sears and Zemansky's University Physics with Modern Physics (14th edition) [M]. Pearson Education. 2015.

[2] David Halliday, Robert Resnick, Jearl Walker. Fundamentals of Physics: Extended (11th Edition) [M]. New York: John Wiley \& Sons, Inc., 2018: 587.

[3] Ulf W. Gedde. Essential Classical Thermodynamics [M]. Springer, 2020.

[4] Stephen R. Turns, Laura L. Pauley. Thermodynamics: Concepts and Applications [M]. Cambridge University Press, 2020.

[5] Jean-Philippe Ansermet, Sylvain D. Brechet. Principles of thermodynamics [M]. Cambridge University Press, 2019.

[6] Ramamurti Shankar. Fundamentals of Physics I: Mechanics, Relativity, and Thermodynamics [M]. Yale University Press, 2019.

[7] Kim Sharp. Entropy and the Tao of Counting: A Brief Introduction to Statistical Mechanics and the Second Law of Thermodynamics [M]. Springer International Publishing, 2019.

[8] Robert H. Swendsen. An Introduction to Statistical Mechanics and Thermodynamics [M]. Oxford University Press, 2019.

[9] Antonio Saggion, Rossella Faraldo, Matteo Pierno. Thermodynamics - Fundamental Principles and Applications [M]. Springer, 2019.

[10] Gregor Skačej, Primož Ziherl. Solved Problems in Thermodynamics and Statistical Physics [M]. Springer, 2019.

[11] Luscombe, James. Thermodynamics [M]. CRC Press, 2018.

[12] M. Tabatabaian, Er. R. K. Rajput. Advanced thermodynamics: fundamentals, mathematics, applications [M]. Mercury Learning \& Information, 2018.

[13] V. Ganesan. Thermodynamics: Basic And Applied [M]. Mc Graw Hill India, 2018.

[14] Wolfgang Demtröder. Mechanics and Thermodynamics [M]. Springer, 2017.

[15] Jochen Rau. Statistical Physics and Thermodynamics: An Introduction to Key Concepts [M]. Oxford University Press, 2017. 
[16] Mungan C E. Entropy change for the irreversible heat transfer between two finite objects [J]. European Journal of Physics, 2015, 36 (4).
[17] Lima F M. Increase of entropy in the thermalization of two blocks: a simpler proof [J]. European Journal of Physics, 2015, $36(6)$. 